\title{
La autopercepción del turista cultural en su rol de turista
}

\author{
Fernando Pellicer* Raquel Camprubí** \\ Universidad de Girona (España)
}

\begin{abstract}
Resumen: La autopercepción del individuo ha sido explorada desde distintas aproximaciones de las ciencias sociales, aunque muy pocos estudios se han centrado en la autopercepción del individuo como turista. A partir del uso de entrevistas semiestructuradas, este trabajo pretende servir de aproximación a la percepción que el turista tiene de sí mismo en un contexto turístico de tipología cultural. Si bien los turistas culturales suelen autopercibirse de manera distinta al resto de visitantes, las actividades que realizan son bastante similares. Los resultados del estudio muestran que la autopercepción del turista cultural está sujeta a múltiples factores, que el hecho de considerarse un turista típico no necesariamente supone un atributo negativo en la percepción individual propia y que el turista cultural se identifica como tal, aunque no de manera inmediata.

Palabras Clave: Turismo cultural; Autopercepción; Autoconcepto; Turista cultural; Girona.

\section{Tourist self-perception in its tourist role}

Abstract: Self-perception of individuals has been explored from various perspectives in the social sciences; although, very few studies have focused on self-perception of individuals as tourists. By using semi-structured interviews, this research aimed at approximating a vision of a tourist's self-perception indestinations defined as cultural. The activities of tourists who visit cultural destinations are in every way similar to other tourists although they perceive themselves differently. Hence, the results of the study show that the cultural tourist' self-perception depends on multiple factors, that it is not necessarily negative for them to perceive themselves as typical tourists but that it is not an immediate self-perception.
\end{abstract}

Keywords: Cultural tourism; Self-perception; Self-concept; Cultural tourist; Gerona.

\section{Introducción}

Según datos de la Organización Mundial del Turismo (OMT), en 2017 se estimó que el turismo cultural se vinculaba con algo más del 39\% de las llegadas internacionales (UNWTO, 2018:21). No obstante, solo un pequeño porcentaje que oscila entre el $5 \%$ y el $10 \%$ de los turistas se llega a identificar como turista cultural (Richards, 2018). Cabe señalar entonces que, en ocasiones, hay discrepancias entre lo que las personas piensan que son y lo que realmente son, especialmente cuando se trata de atributos negativos (Prebensen et al., 2003). Según Culler (2007), ser turista constituye un aspecto negativo en la percepción individual propia, de modo que los turistas no quieren ser vistos como turistas sino como viajeros.

Son muchas las definiciones existentes sobre la autopercepción que se pueden encontrar en la literatura académica de las ciencias sociales, siendo mayoritariamente las tradicionales aquellas que presentan un enfoque individualista mientras que las más recientes hablan de la existencia de múltiples personalidades (Sirgy y Su, 2000). De hecho, numerosos estudios confirman que el consumo es un vehículo de autoexpresión que lleva al cliente a elegir los productos y marcas que percibe como similares al concepto que tiene de sí mismo (Hosany, 2016). En materia turística se ha demostrado que la congruencia de la autoimagen tiene una gran influencia en la toma de decisiones del destino a visitar (Phucharoen et al., 2016).

Por otra parte, las diferencias entre los distintos tipos de turistas ha sido un tema central de los estudios turísticos (McCabe, 2005). De hecho, las investigaciones relacionadas con el turismo cultural

\footnotetext{
* Universidad de Girona (España); E-mail: fernandopellicer95@gmail.com

** Universidad de Girona (España); E-mail: raquel.camprubi@udg.edu; https://orcid.org/0000-0001-7084-7889
} 
han prestado gran atención a la identificación de distintos tipos de perfiles a través de enfoques y perspectivas muy diversas, siendo las motivaciones de los visitantes una forma comúnmente utilizada para clasificar a los turistas culturales (Richards, 2018). Similarmente, la tipificación de segmentos turísticos en función de los estilos de vida de los usuarios ha sido una práctica usada para aproximarse de mejor manera a la comprensión del comportamiento de los turistas (Swarbrooke y Horner, 2007). Sin embargo, según Todd (2001), esta forma de segmentar no tiene en consideración el cómo se siente la persona en el momento en el que se está llevando a cabo la actividad, habiendo muy pocos estudios acerca de cómo se perciben los individuos en su rol de turista. Eso resulta especialmente interesante si se tiene en cuenta que la gente viaja para escapar de las rutinas establecidas de su espacio habitual, desplazándose a nuevos lugares y entornos que conllevan que el individuo cambie su modo normal de comportamiento (Boissevain, 2005).

Con todo ello, el objetivo principal de este trabajo es aproximarse a la autopercepción del turista cultural. Esto no solamente implica el cómo se percibe éste a sí mismo y si se considera un turista cultural o no, sino también tratar de identificar qué factores son los que configuran su propia imagen, entender cuáles son las diferencias que estos turistas encuentran entre ellos y el resto de visitantes, y verificar si el hecho de ser un turista se percibe realmente de manera negativa. Debido a que son varios los estudios que tratan el tema de la autopercepción en el ámbito turístico de manera cuantitativa (Todd, 2001; Prebensen et al., 2003; Doran et al., 2015; Hosany, 2016; Yu et al., 2012), esta investigación pretende responder a estas cuestiones a partir de un enfoque cualitativo aplicado a un contexto de destino turístico cultural, tomando la ciudad de Girona como caso de estudio.

\section{Marco teórico}

\subsection{El turismo y el turista cultural}

Se entiende por turismo cultural la actividad turística en la cual el motivo principal del viaje es el afán por aprender, descubrir y experimentar los distintos productos y atractivos culturales tangibles e intangibles de un destino (Richards, 2018). Ésto incluye el acercamiento a una serie de manifestaciones materiales, intelectuales, espirituales y emocionales que presenta una sociedad a través de múltiples aspectos como puede ser su patrimonio histórico y cultural, su arquitectura, arte, gastronomía, tradiciones y estilos de vida, entre otros (UNWTO, 2017:18). De este modo se deja de vincular el turismo cultural al mero disfrute de monumentos y museos, para relacionarlo con la tendencia creciente de que el turista busca la experiencia original y vivir la realidad del destino (Yeoman et al., 2007). Sin embargo, no todos los turistas culturales presentan las mismas motivaciones ni intereses por el mismo tipo de vivencias (McKercher, 2002), habiendo múltiples formas de catalogar a éstos.

Una de las clasificaciones más aceptadas es la elaborada por Richards (1996) que diferencia entre el turista cultural "general" y el "específico" en función de si el consumo de cultura forma sólo una parte de las vacaciones o si, en cambio, ésta es el motivo principal del viaje. Silberberg (1995), por su parte, identifica cuatro tipos de turistas culturales. Así pues, los clasifica en "altamente motivados" o "motivados en parte" por la cultura según el nivel de importancia que los turistas dan al aspecto cultural de la visita a un destino. A su vez también identifica a los turistas "adjuntos" que perciben en la cultura una motivación secundaria; mientras que el último grupo es el conformado por los turistas culturales "accidentales" que son aquellos que asisten a atracciones culturales sin realmente haberlo planeado. De forma similar, Hughes (2002) determina dos grandes grupos también en función de la importancia que los turistas dan a los elementos culturales. Por un lado, identifica a los turistas "culture-core" que son aquellos para los cuales la cultura es el núcleo de la visita y dentro de esta categoría diferencia a los "primarios" de los "multiprimarios". Por otro lado, reconoce a los "culture-peripheral" que son aquellos turistas que no dan tanto peso al aspecto cultural en su desplazamiento al destino y en cuyo grupo identifica a los "incidentales" de los "accidentales". Basándose en la centralidad y la profundidad de la experiencia, McKercher (2002) distingue cinco tipos de turistas culturales: los decididos, los observadores, los casuales, los incidentales y los fortuitos. Según McKercher y du Cros (2003), dicha clasificación guarda cierta relación con la manera en la que Plog clasifica a los turistas a partir de sus características psicográficas. Así pues, Plog (1976) identifica dos grandes grupos de turistas. En un extremo sitúa al turista "psicocéntrico" el cual es algo reacio a viajar, hace estancias breves y se limita a visitar destinos maduros para conocer solamente sus principales atractivos, mientras que en el otro extremo se encuentra el turista "alocéntrico" que se caracteriza por hacer largos viajes en destinos descono- 
cidos donde intenta mezclarse con los locales. Entre estos dos perfiles, Plog (1976) identifica varios escenarios intermedios entre los cuales destaca la figura del turista "mediocéntrico" por ser la más común entre los visitantes según el racionamiento de este autor. Este tipo de turista se caracteriza por no ser excesivamente arriesgado en sus viajes, aunque sí muestra cierta disposición a vivir nuevas experiencias. Por consiguiente, la clasificación del turista de McKercher (2002) se vincula con esta agrupación psicográfica realizada por Plog en cuanto que los turistas culturales decididos se asemejan en motivaciones a los turistas alocéntricos, los observadores a los mediocéntricos, en tanto que los tres restantes se corresponden con los psicocéntricos (McKercher y du Cros, 2003). Otra clasificación del turista es la planteada por Galí y Donaire (2006) en un estudio que tuvo como campo de investigación la ciudad de Girona. Éstos identificaron cuatro tipos de turistas en función de la relación que los visitantes presentaban con el patrimonio de la localidad, siendo los turistas rituales, los no culturales, los interesados y los eruditos los grupos establecidos en dicho estudio.

Con todo, identificar quién es un turista cultural es algo complejo, no solamente porque se aplica a las visitas de una amplia y diversa gama de atracciones y el hecho que los motivos de visita a los destinos suelen ser difusos e interrelacionados (Hughes, 2002; Niemczyk, 2013), tal y como se ha argumentado; sino también debido a la actual inmersión en una sociedad basada en el concepto de liquidez de Bauman (2000). El movimiento constante y la falta de permanencia son a vistas de este autor la principal característica de la sociedad moderna actual, en la que las fronteras que definen al individuo se difuminan, y en la que todos podemos ser o no ser turistas en función del tiempo y el espacio (Bauman, 1996; 2000).

\subsection{El antiturismo como contraposición al turista típico}

Pese a las múltiples clasificaciones existentes acerca de la figura del turista cultural, McKercher (2004) llega a la conclusión de que éstos tienden a participar en el mismo tipo de actividades y atracciones culturales independientemente del país o lugar de destino. Esto puede resultar paradójico si se tiene en cuenta que en los años noventa ya múltiples autores afirmaban la existencia de una tendencia creciente por la búsqueda de viajes individualizados en contraposición a los tradicionales paquetes turísticos, siendo ésta una característica del turismo contemporáneo (Osti et al., 2009). Así pues, el impacto de las nuevas tecnologías y la contribución de las mismas en el proceso de globalización del conocimiento, han permitido que las personas puedan dibujar su identidad a través del acceso a productos y servicios que satisfagan sus necesidades individuales (Yeoman et al., 2007). Esto deriva del significado que las personas otorgan a los distintos tipos de bienes, servicios o marcas, siendo el consumo un medio de expresión que sirve al consumidor para diferenciarse del resto (Sirgy, 1982). En turismo, la recreada necesidad que tienen algunos turistas de distanciarse de sus iguales se denomina antiturismo (Jacobsen, 2000), motivo por el cual en muchas ocasiones el turista intente aproximarse al local y trate de ser "uno de ellos" puesto que eso implica una mayor autenticidad. No obstante, para Bauman (Franklin, 2003) esto no es nada más que una paradoja, ya que según su razonamiento la posibilidad de tener un encuentro genuino es más probable cuando el individuo se queda en casa (en grandes ciudades) que cuando viaja a miles de kilómetros de distancia de su residencia.

Según MacCannell (1973) el término "turista" se vincula cada vez más con experiencias de contenidos poco auténticos. Festinger (1954) sostiene que las personas presentan una clara tendencia en comparar sus propias habilidades y opiniones con la del resto porque existe una necesidad inherente de la autoevaluación. Es en esta comparativa en la cual los turistas acaban afirmando que su perfil no se corresponde con el del turista típico incluso cuando las actividades realizadas son las mismas (Prebensen et al., 2003). Doran et al. (2015) confirma que los turistas dicen diferenciarse del turista típico en términos de motivaciones. Con todo, se considera por típico el rol del turista que participa en tours guiados y viajes organizados (Jacobsen, 2000). De hecho, Pizam y Sussmann (1995) elaboran una lista enumerando veinte características comúnmente asociadas a la figura del turista típico y entre las cuales están el hacer fotos, comprar suvenires o querer ver "la parte real" del destino. En contraposición, los turistas son más propensos a identificar sus propias experiencias y actividades en categorías que consideran menos ofensivas a la vinculada al concepto "turista" (McCabe, 2005), evitando afirmar que visitan algunos espacios porque son populares o atraen mucho turismo (McCabe y Stokoe, 2004). Todo ello conforma un paradigma en el cual los turistas tratan de huir de lo turístico (Richards y Russo, 2016), siendo la denigración feroz de los turistas un intento de convencerse de que uno no forma parte de ese grupo (Culler, 2007). 


\subsection{El autoconcepto en materia turística}

El autoconcepto es la totalidad de pensamientos y sentimientos que un individuo referencia sobre sí mismo (Rosenberg, 1979:7). Sin embargo, la percepción de la propia persona no es constante sino que varía en función de la situación en la que ésta se encuentre (Todd, 2001). El turista en el viaje se enfrenta a un cambio de rutina puesto que realiza un desplazamiento hacia un espacio y contexto distinto al de su vida cotidiana, cambiando así su forma habitual de comportarse (Cohen, 1974; Boissevain, 2005). De este modo, el autoconcepto debe entenderse desde un punto de vista multidimensional que depende de variables personales y situacionales (Malhotra, 1988), teniendo la persona tantos seres sociales como individuos que la reconocen y crean una imagen de la misma en su mente (James, 1950:254). Es por ello que Sirgy (1980) considera la existencia de cuatro perspectivas del autoconcepto. Por un lado, identifica la "autoimagen actual" como el grado en el cual un sujeto cree que tiene una imagen específica, diferenciándose de la "autoimagen social" en cuanto que ésta se relaciona con el hecho de que el individuo percibe que los otros tienen una imagen de él. Por otro lado, este autor define a la "autoimagen ideal" como el grado en el cual un sujeto quisiera tener una imagen concreta, siendo finalmente la "autoimagen social ideal" aquella que a la persona le gustaría que los otros percibieran de sí misma. Esto revela el papel que tiene la opinión de terceros en la determinación del autoconcepto de un individuo.

Además de todo esto, Yu et al. (2012) señala que en materia turística la lejanía condiciona la autopercepción del sujeto, habiendo una correlación positiva entre la distancia recorrida y la probabilidad de que un visitante se considere turista. Similarmente, la repetición también es un factor influyente en el autoconcepto del turista, observándose una mayor tendencia a identificarse como tal entre las personas que visitan un destino por primera vez y no tanto entre aquellas que han estado con anterioridad (Woosnam et al., 2018). Esto generalmente se atribuye al hecho de que los que viajan por primera vez suelen hacerlo más interesados en conocer un lugar, mientras que los repetidores se desplazan por motivos de recreación, enfocados en una actividad muy concreta o a visitar amigos y familiares (Yu et al., 2012). Asimismo, Hosany (2016) afirma que, en cierto tipo de experiencias turísticas, el nivel de congruencia existente entre la imagen propia de un turista con la que éste tiene sobre los otros turistas, afecta en el grado de satisfacción global. De hecho, en turismo el autoconcepto ha sido utilizado frecuentemente en el estudio de la congruencia de la imagen (Beerli et al., 2007), demostrándose a través de la investigación empírica que cuanto mayor es el acuerdo entre la imagen de un destino y el autoconcepto, mayor es la tendencia del turista a visitar dicho lugar (Sirgy y Su, 2000). A pesar de ello, son muy pocos los trabajos llevados a cabo para entender cómo se perciben los sujetos en su rol de turista (Doran et al. 2015), siendo el objetivo principal de este estudio el realizar una aproximación a la percepción y los sentimientos que el turista cultural tiene sobre sí mismo en el momento en el que está realizando el viaje.

\section{Metodología}

\section{1. Área de estudio}

Girona es una ciudad española situada en el noreste de la Península Ibérica, a escasos sesenta kilómetros de la frontera francesa. Su casco antiguo presenta una gran cantidad de elementos patrimoniales que configuran un marco de alto valor histórico y artístico. En los últimos años, la ciudad ha consolidado su estrategia turística convirtiéndose así en un importante centro de turismo cultural (Galí et al., 2015). Según Galí y Donaire (2006), Girona es considerada un arquetipo de los lugares que representan la cultura europea en España, por lo que este trabajo se enmarca en un contexto y espacio caracterizado por su valor histórico y monumental.

\subsection{Población y muestra de estudio}

La población a investigar en este estudio está constituida por todos los turistas que visitan la ciudad de Girona. Esto, según la definición de la OMT, implica que solamente formaron parte de la población aquellos visitantes que pernoctaron en dicha ciudad al menos una noche (UNWTO, 2008).

La técnica de muestreo empleada fue la del muestreo por conveniencia que, según Mejía (2000), es un procedimiento de muestreo comúnmente utilizado en las investigaciones de carácter cualitativo en el que los informantes son seleccionados en función de su fácil disponibilidad y acceso. Los únicos criterios que se tuvieron en cuenta a la hora de elegir a los entrevistados fue que el informante pernoctase al menos una noche en la ciudad de Girona durante su estancia, y que no estuviese realizando una actividad organizada contractualmente en el momento en el que se hizo la entrevista. La muestra seleccionada para 
el desarrollo de este trabajo estuvo formada por 30 turistas de diferentes edades y procedencias (tabla 1 y 2). Según Bertaux (1993) el nivel de saturación se consigue a partir de los 30 casos, es decir, el punto en el cual introducir más participantes en la investigación no aporta nada nuevo (Bowen, 2008). En este caso se empezó a apreciar la reiteración de ideas a partir de la entrevista número veinticinco, aunque se hicieron cinco más para asegurar dicho nivel de saturación. La interceptación de los participantes fue llevada a cabo en seis de los puntos gerundenses más frecuentados por la actividad turística: las escaleras de la Catedral de Girona, la Plaza de los Apóstoles, el Paseo Arqueológico, los Jardines de los Alemanes, la Rambla de la Libertad y las escaleras de la Basílica de San Félix, entre los días 30 de abril y 09 de mayo de 2019 .

Tabla 1: Perfil sociodemográfico de la muestra

\begin{tabular}{|c|c|c|}
\hline \multicolumn{1}{|c|}{ Variables } & Frecuencia & \\
\hline Sexo & 18 & 60 \\
\hline Hombre & 12 & 40 \\
\hline Mujer & 15 & 50 \\
\hline Edad & 9 & 30 \\
\hline$<30$ & 6 & 20 \\
\hline $30-50$ & & \\
\hline$>50$ & 7 & 23,3 \\
\hline Origen & 3 & 10 \\
\hline Nacional & 4 & 13,3 \\
\hline Cataluña & 23 & 76,7 \\
\hline España & 15 & 50 \\
\hline Extranjero & 4 & 13,3 \\
\hline Luropa & 4 & 13,3 \\
\hline Otros & & \\
\hline
\end{tabular}

Tabla 2: Caracterización y perfil sociodemográfico de los entrevistados

\begin{tabular}{|c|c|c|c|c|}
\hline $\begin{array}{c}\text { Código } \\
\text { entrevista }\end{array}$ & Sexo & Procedencia & Edad & Repetición \\
\hline E1 & F & Bélgica & $30-50$ & Sí \\
\hline E2 & M & Israel & $<30$ & No \\
\hline E3 & F & Cataluña & $<30$ & Sí \\
\hline E4 & M & España & $<30$ & No \\
\hline E5 & M & Holanda & $30-50$ & No \\
\hline E6 & F & Argentina & $30-50$ & Sí \\
\hline E7 & M & Cataluña & $<30$ & No \\
\hline E8 & M & Italia & $<30$ & No \\
\hline E9 & F & Chile & $30-50$ & $30-50$ \\
\hline E10 & F & Francia & & \\
\hline
\end{tabular}




\begin{tabular}{|c|c|c|c|c|}
\hline $\begin{array}{c}\text { Código } \\
\text { entrevista }\end{array}$ & Sexo & Procedencia & Edad & Repetición \\
\hline E11 & M & Hawái & $>50$ & No \\
\hline E12 & M & Rusia & $<30$ & No \\
\hline E13 & M & China & $<30$ & No \\
\hline E14 & M & Cataluña & $30-50$ & Sí \\
\hline E15 & M & Estonia & $<30$ & No \\
\hline E16 & $\mathrm{F}$ & Francia & $<30$ & Sí \\
\hline E17 & M & Reino Unido & $<30$ & No \\
\hline E18 & M & Francia & $30-50$ & Sí \\
\hline E19 & $\mathrm{F}$ & Argentina & $>50$ & No \\
\hline E20 & $\mathrm{F}$ & Holanda & $>50$ & No \\
\hline E21 & M & Portugal & $>50$ & No \\
\hline $\mathrm{E} 22$ & $\mathrm{~F}$ & Polonia & $<30$ & No \\
\hline E23 & $\mathrm{F}$ & España & $<30$ & No \\
\hline E24 & M & Alemania & $>50$ & No \\
\hline E25 & M & Letonia & $<30$ & No \\
\hline E26 & $\mathrm{F}$ & Reino Unido & $30-50$ & No \\
\hline E27 & M & Cataluña & $<30$ & No \\
\hline E28 & $\mathrm{M}$ & España & $>50$ & No \\
\hline E29 & $\mathrm{F}$ & Argentina & $30-50$ & No \\
\hline E30 & M & Italia & $<30$ & No \\
\hline
\end{tabular}

\subsection{Recogida y análisis de datos}

Con el objetivo de obtener respuestas abiertas, se empleó el uso de entrevistas semiestructuradas previamente testadas. Éstas, según Barriball y While (1994), resultan especialmente adecuadas para explorar las percepciones y opiniones de los participantes, sobre todo cuando la muestra presenta características personales muy dispares. De esta forma, todas las entrevistas realizadas partieron de un guión preestablecido el cual se configuró a partir de la teoría de la autopercepción planteada por Sirgy (1980). Con todo, la función principal de dicho guión fue la de dirigir la conversación entre el investigador y el informante, ejerciendo así de material de apoyo para asegurar el tratamiento de todos los temas de interés en cada una de las entrevistas. La estructura marcada, por tanto, no se cumplió de manera estricta en todas ellas, sino que se las dotó de cierta flexibilidad. Sin embargo, el proceso general de recopilación de datos comenzaba preguntando al informante por las motivaciones de su visita en Girona, los medios y canales de información utilizados, la planificación, expectativas y actividades realizadas. Una vez comentados estos aspectos, se le pedía al entrevistado que tratase de concretar qué tipo de turista se consideraba para luego preguntarle si realmente era ese el turista que le gustaba ser. Tras profundizar un poco más en el tema, se le planteaba al informante el hecho de poder considerarse a sí mismo el típico turista, pues a partir de su respuesta se le pedía que intentase explicar cuáles eran las características y actividades que él vinculaba al turista típico que visitaba Girona e hiciese una breve comparativa con lo que había hecho hasta el momento. Seguidamente, se le preguntaba al entrevistado cómo creía que era percibido por el resto en su rol de turista y si prefería que éstos le viesen de un modo distinto. Por último, cabe señalar que al final de las entrevistas se le solicitaba al informante que justificase si le molestaba poder ser visto como el típico turista y también si se llegaba a considerar un turista cultural en la ciudad gerundense. 
Para el análisis de los datos obtenidos y la correspondiente extracción de conclusiones se ha empleado la metodología de análisis de contenido de carácter cualitativo. Se trata de una técnica cada vez más utilizada en investigaciones turísticas desarrolladas a partir de entrevistas (Camprubí y Coromina, 2016). Esta técnica supone una aproximación empírica a través del análisis metodológico y detallado de textos producidos en un contexto de comunicación concreto (Mayring, 2000). Es por ese motivo que las entrevistas fueron grabadas y cuidadosamente transcritas, identificando posteriormente patrones comunes entre los informantes, pero también prestando atención a las particularidades de sus respuestas como bien señala Elo y Kyngäs (2008). Según Cáceres (2008), uno de los usos de esta metodología es la de integrar los datos obtenidos a interpretaciones de nivel superior que permitan establecer relaciones entre los puntos de estudio con la teoría existente, tal y como queda recogido en los resultados que se presentan en el siguiente apartado.

\section{Resultados}

\subsection{La autoimagen actual y la autoimagen ideal del turista}

Son muchas las motivaciones que llevan a los turistas a emprender un viaje (Richards, 2018). Tras el análisis del contenido de las entrevistas realizadas, se afirma que éstas tienen una gran influencia a la hora de constituir la autopercepción del turista. "Conocer una ciudad nueva, relajarse, pasar unos días agradables, cambiar de ambiente..." Estos fueron algunos de los ejes fundamentales de las respuestas a la pregunta ¿qué tipo de turista te consideras? Sin embargo, cuando se les pidió a los informantes que se definiesen como turistas, sus respuestas resultaron asociarse a rasgos del perfil psicográfico identificados por Plog (2001). Así pues, resaltaron no sólo características personales como lo son la curiosidad o la organización, sino también aspectos vinculados con la forma de viajar tales como lo son los conceptos de turista mochilero, turista independiente, turista de paso o turista que intenta ser un local.

Asimismo, se encontraron otros tres factores determinantes a la hora de definir la autopercepción del turista. Dos de ellos son la distancia y la repetición, elementos ya identificados por Yu et al. (2012) y Woosman et al. (2018). Con ello, cabe destacar la expresión "no me considero tan turista ya que vivo a escasos 80 kilómetros" (E18) por parte de un visitante francés que no solamente había cambiado de país, sino que pernoctaba dos noches en la ciudad catalana. Similarmente, entre los repetidores era bastante común escuchar aquello de "este fin de semana no venimos tanto a visitar sino a pasear"(E14) o "hemos estado varias veces en Girona y ya nos sentimos como en casa. La primera vez fue distinto, mucho más de hacer turismo" (E1). El tercer elemento es el acompañante, pues aquellos entrevistados que se encontraban haciendo la visita acompañados de algún residente afirmaron sentirse menos turistas al considerarse en mayor contacto con la gente local. En consecuencia, y pese a considerarse turista, una participante manifestaba lo siguiente: "creo que me alejo un poco del concepto turista en cuanto que me dejo llevar por ella [su amiga]. He estado en calles y bares por los que los turistas no pasan. Voy a quedar con gente de aqui” (E3).

Todo lo hasta ahora mencionado trata de configurar la autoimagen actual descrita por Sirgy (1980) en la figura del turista. Independientemente de cuál fuese ésta, se encontró pleno consenso en cuanto que todos los entrevistados afirmaron estar contentos siendo el tipo de turista que ellos creían ser, con la única excepción de una participante que expresaba su deseo por ser una turista algo más organizada. Este hallazgo indica que suele existir un gran ajuste entre la autoimagen actual e ideal del turista cultural. Ahora bien, ¿puede estar esto relacionado con que los turistas culturales realmente se perciben a sí mismos como turistas no típicos?

\subsection{Turista típico vs Turista no típico}

Algunas personas son reacias a ser vistas como turistas cuando están viajando (Jacobsen, 2000). Esto coincide con los resultados de Prebensen et al. (2003) en el que un 89'5\% de los encuestados afirmaba no ser el típico turista alemán que visitaba Noruega. Según Culler (2007), este hecho se debe a que el concepto turista comporta connotaciones negativas en la percepción del individuo. En este caso de estudio el fenómeno volvió a repetirse, encontrándose a una gran mayoría de los entrevistados que afirmaron no considerarse el típico turista. Los participantes justificaron ese sentimiento no tanto en las actividades y nodos de visita, sino en la forma de viajar, algo ya señalado por Prebensen et al. (2003). Con todo, se atribuyó al turista típico que visita Girona características como una notable preparación y organización del viaje, preferencia por la realización de tours guiados y estancias de corta duración que normalmente no implican dormir en la ciudad. Así lo manifestaba un entrevistado al decir que "el 
turista típico que visita Girona, en mi opinión, se aloja en Barcelona y simplemente pasa unas horas en esta ciudad. Tienen que venir con todo organizado para poder visitarlo todo" (E11). Por otra parte, el hecho de que los participantes utilizasen el término turista para referirse a visitantes que no pernoctan en el destino, confirma lo apuntado por Yu et al. (2012) en cuanto que la distinción entre turista y excursionista es principalmente técnica y que, por tanto, no acaba de ser asimilada entre la población general. De este modo, los entrevistados se distanciaron del concepto del turista típico alegando que ellos viajaban sin prácticamente haber planificado nada, siendo un tipo de turista individual e independiente cuya libertad le permitía visitar todos los atractivos que verdaderamente llamasen su atención, sin las limitaciones de ningún recorrido impuesto. "Ellos reservan tours, excursiones, actividades... Todo preparado. Y nosotros hemos venido a la aventura" (E2). Igualmente, de manera similar a lo afirmado por MacCannell (1973), fueron muchos los participantes que emplearon su interés de vivir experiencias locales como elemento de diferenciación entre ellos y el resto de turistas. En esto hicieron mayor énfasis los más jóvenes, los que hicieron estancias más largas, los repetidores y los acompañados por residentes.

No obstante, y pese a que en su autoimagen actual los participantes no se concibieron como turistas típicos, éstos no manifestaron sentirse molestos en caso de ser vistos como tal por el resto. "¿Cómo me va a molestar que me identifiquen con una turista si el turismo me parece algo maravilloso, que disfruto y que conlleva un aprendizaje?" (E29). De hecho, algunas de las respuestas fueron llamativas en cuanto que expresaban que ser un típico turista no significaba nada malo en la percepción propia sino más bien que dicha negatividad se vinculaba con el concepto que el resto pudiese tener acerca de lo que es un turista típico. Un ejemplo de ello fue la respuesta de un participante italiano que textualmente dijo: "en un principio no me molesta, aunque depende de lo que la gente considere por típico turista. Si implica que no voy a respetar a la gente ni a la ciudad, pues obviamente me molesta que me vean asi" (E8). Visto de esta forma, resulta bastante evidente que en la formación de la autopercepción del turista interviene la percepción que los otros tienen del mismo (Sirgy y Su, 2000), especialmente en un contexto en el que el turista se encuentra de manera temporal y en el cual no percibe la necesidad de seguir las normas y pautas establecidas, tal y como determina Bauman (Franklin, 2003).

\subsection{La autoimagen social y la autoimagen social ideal del turista}

En la mayoría de los estudios de consumo se ha prestado atención solamente a la autoimagen actual e ideal del individuo (Hosany, 2016). Sin embargo, Sirgy (1980) defiende que toda autopercepción está en cierto modo condicionada a la opinión de terceros. En este caso de estudio, resultó interesante observar cómo los informantes confirmaban tener la sensación de ser percibidos como el típico turista pese a ellos no identificarse con este término. Según los participantes, esta imagen se transmitía a partir de aspectos visibles como el simple hecho de llevar un mapa, una mochila o una cámara de fotos; coincidiendo así con algunas de las características atribuidas al típico turista por Pizam y Sussmann (1995). Los entrevistados extranjeros añadieron, además, que existía una alta probabilidad de que se les considerase un turista típico simplemente por su forma de vestir, su color de piel o el idioma hablado. Sin embargo, esta autoimagen social quedó dividida en dos partes. Por un lado, la imagen percibida por el resto de turistas y, por otro, la interpretada por los locales. La primera de ellas fue algo más generalista en cuanto que, según los entrevistados, entre turistas todos acaban percibiéndose de igual a igual. La segunda, en cambio, sí acarreaba connotaciones negativas ante la posibilidad de ser percibidos por los locales como un estorbo. "Yo creo que algunos de los que viven aquí me ven mal, como que les molesto e interrumpo su paso... Incluso creo que pueden llegar a pensar que por mi culpa todo es más caro" (E6) fueron las palabras de una entrevistada argentina. Esto se relaciona con el hecho de que a medida que una ciudad depende cada vez más del sector turístico, los efectos negativos son más reconocibles que los positivos entre la población local (McGehee y Andereck, 2004). Con todo, esta sensación de ser percibido como un turista típico por el resto no fue expresada por los visitantes de proximidad, los repetidores o los acompañados por residentes en Girona.

Siguiendo las aportaciones de Sirgy (2000), el último de los componentes que conforman la autopercepción del turista es la autoimagen social ideal. Para ello se le preguntó a los entrevistados si preferían ser vistos de otra forma una vez planteada la cuestión de cómo creían ser percibidos por el resto en su rol de turista. Ante esta pregunta los informantes mostraron dar poca importancia al hecho de ser vistos de una forma u otra, asumiendo muchos de ellos que les parecía normal que se les percibiese como turistas porque esa imagen se correspondía con lo que en ese momento eran. "Para nada me importa que me vean como el típico turista. Yo sé que lo parezco" (E17) por parte de un entrevistado inglés, al igual que la respuesta de una visitante chilena al decir "estoy haciendo turismo y soy otra turista más. 
Es normal que me vean así y no me molesta en absoluto" (E9) son solamente algunos ejemplos de ello. Sin embargo, se expresó el deseo de no querer ser visto como algo molesto entre los residentes por parte de aquellos turistas que contemplaron la posibilidad de que el turismo pudiese incomodar a la población local, como bien señalan algunos autores (McGehee \& Andereck, 2004; Woosnam et al., 2018).

\subsection{La autopercepción del turista cultural}

El turismo cultural representa una parte considerable de las llegadas internacionales (UNWTO, 2018), y aunque son muchas las definiciones y clasificaciones que se hacen del turista cultural (Richards, 1996; Silberberg, 1995; Hughes, 2002; McKercher, 2002; Galí y Donaire, 2006), solamente se identifican como tal entre el 5\% y el 10\% (Richards, 2018). Teniendo en cuenta que Girona es un importante centro de turismo cultural (Galí et al., 2015), cabría esperar que una gran proporción de los entrevistados fuesen, en alguna de todas sus acepciones, turistas culturales. Si bien es cierto que muchos de ellos afirmaron encontrar entre sus motivaciones principales para viajar el hecho de conocer la cultura e historia de la ciudad de Girona, ninguno de ellos se identificó directamente como un turista cultural cuando se le preguntó por el tipo de turista que era. A pesar de ello, al final de la entrevista se planteaba a los participantes si llegaban a considerarse un turista cultural en la ciudad catalana, obteniendo un sí como respuesta en la gran mayoría de las ocasiones. No lo hicieron tanto aquellos que ya habían estado con anterioridad en Girona. Igualmente, hubo algunos casos aislados que respondieron que todavía no se consideraban turistas culturales porque no habían llegado a visitar apenas nada en el momento en el que se estaba realizando la entrevista, apuntando que esta sensación cambiaría una vez visto todo aquello que tenían en mente. Esto implica que, en cierta medida, el autoconcepto de ser un turista cultural o no está sujeta tanto a un factor temporal, como a los elementos y espacios visitados.

Además de ello, cabe destacar que no todos los que dijeron considerarse un turista cultural lo hicieron por los mismos motivos, otorgando diversos niveles de importancia a los elementos culturales, tal y como señalan las clasificaciones de Silberberg (1995) o la de Hughes (2002) anteriormente citadas. En consecuencia, se encontraron posturas del tipo "me considero turista cultural porque disfruto viendo los monumentos, aunque tampoco voy a buscar información [sobre los monumentos]" (E2), "lo sería a medias ya que mi objetivo principal es despejarme, aunque está claro que estoy viendo cosas culturales y me están gustando" (E6), "yo creo que sería semicultural porque no voy a poder abarcar todo lo que la ciudad ofrece" (E28) o "he venido sobre todo por la cultura, de ahi que haya estado dentro de la Catedral, de la Basílica de San Félix, de los Baños Árabes y en el Museo de Historia”(E10).

En términos generales, también se le preguntó a los entrevistados si a la hora de elegir un destino al que visitar tenían en consideración que éste ofreciese atracciones y elementos culturales. De manera prácticamente unánime los participantes contestaron que sí, mostrando nuevamente matices muy distintos en sus respuestas. Con ello, algunos manifestaron que la presencia de estos elementos era imprescindible porque cuando viajan les gusta ver "cosas y monumentos", apuntando que éstos consiguen que un viaje sea mucho más sencillo de estructurar en el sentido de que saben qué es lo que tienen que ir a visitar. Para otros la importancia residía en que a partir de estos atractivos podían llegar a entender la cultura y la historia de la ciudad que estaban visitando y la forma de pensar y actuar de sus habitantes.

Así pues, pese que de primeras los turistas no lleguen a identificarse de manera inmediata como turistas culturales (Richards, 2018), este estudio llega a la conclusión de que éstos sí se consideran como tal, pues el autoconcepto del turista cultural reside en la reflexión que lleva al sujeto a darse cuenta de que los elementos culturales constituyen, de una manera u otra, a formar parte de manera más o menos destacada en su viaje.

\section{Conclusiones}

El turismo implica un cambio en el espacio habitual del individuo que rompe su rutina diaria y modifica su forma normal de comportamiento (Cohen, 1974; Boissevain, 2005). A pesar de ello, la literatura académica ha prestado poca atención a cómo se percibe la gente en su rol de turista (Todd, 2001; Doran et al., 2015). Teniendo en cuenta que la autopercepción de un individuo no es constante y que depende de variables personales y situacionales (Malhorta, 1988), el presente estudio ha tratado de aproximarse a la concepción que el turista cultural tiene de sí mismo atendiendo a los cuatro componentes que según Sirgy (1980) configuran la autopercepción: la autoimagen actual, la autoimagen ideal, la autoimagen social y la autoimagen social ideal. 
De este modo, constituyen la autoimagen actual del turista cultural no tanto las actividades realizadas por éste sino más bien las motivaciones que le llevan a emprender el viaje, sus características psicográficas, la distancia entre el destino y el lugar de origen, el haber estado con anterioridad o no en el sitio visitado y el posible hecho de ir acompañado por un residente. Independientemente de cuál sea esta autoimagen actual, los turistas culturales no suelen manifestar deseo por cambiarla, existiendo así un ajuste entre ésta y su autoimagen ideal. Este acuerdo entre ambas dimensiones de la autopercepción no necesariamente se debe a que los turistas culturales acaben viéndose a sí mismos como turistas no típicos puesto que, en contra de lo afirmado por Culler (2007), ser turista no constituye un aspecto negativo en la percepción individual propia, sino que dicha negatividad se relaciona con la creencia de que el resto pueda considerar que ser un turista típico es algo peyorativo. Por consiguiente, la opinión de terceros influye en el proceso de formación de la autopercepción. Con ello, y de manera opuesta a lo determinado por la autoimagen actual, el visitante es consciente de que tanto locales como turistas le perciben como un turista típico. Esta autoimagen social solamente pretende ser cambiada cuando el sujeto considera que el turismo puede molestar a los residentes del lugar visitado, pues de lo contrario parece existir otro ajuste entre la autoimagen social y la autoimagen social ideal en cuanto que el turista cultural no manifiesta ningún interés por ser visto de otra forma que no sea la de ser un turista más.

Con el objetivo de verificar lo señalado por Richards (2018) respecto a que solo un pequeño porcentaje de turistas llega a identificarse como turista cultural en un momento en el que el turismo cultural se relaciona con aproximadamente el 40\% de las llegadas internacionales (UNWTO, 2018), este estudio enmarca su área de investigación en la ciudad de Girona, un destino de tipología cultural (Galí et al., 2015). Con todo, este trabajo llega a la conclusión de que el turista cultural sí llega a percibirse como tal aunque no de manera inmediata, pues el visitante es conocedor de que los elementos culturales, de algún modo, forman parte de su viaje. Este hecho conlleva a que el individuo pase a considerarse un turista cultural en mayor o menor grado, pudiéndose clasificar así en alguna de las múltiples catalogaciones existentes en torno a la figura del turista cultural (Richards, 1996; Silberberg, 1995; Hughes, 2002; McKercher, 2002; Galí y Donaire, 2006).

Por todo ello, este estudio contribuye a la literatura aportando mayores datos y conocimientos sobre la autopercepción del turista cultural. Además, esta investigación ha sido llevada a cabo a partir de técnicas cualitativas y teniendo en cuenta la influencia de la llamada dimensión social en la creación del autoconcepto, un punto escasamente analizado en los estudios turísticos. Sin embargo, es el propio contexto del caso examinado una limitación en sí mismo en cuanto que no permite confirmar que los hallazgos identificados sean aplicables a la autopercepción de turistas que visitan otros tipos de destinos tales como espacios naturales, parques temáticos, entre otros. Es por este motivo que futuras investigaciones deberían tratar de aproximarse a la autopercepción de otras tipologías de turistas y también de los excursionistas al ser éstos un tipo de visitante con características muy particulares.

\section{Bibliografía}

Ahn, T. H. \& Hosany, S. (2017). The Effects of Self-image Congruence, Tourists' Experiences and Satisfaction on Behavioral Intentions. Asia-Pacific Journal of Business, 8(1), 1-13.

Barriball, L. K. y While, A. (1994. Collecting data using a semi-structured interview: A discussion paper. Journal of Advanced Nursing, 19, 328-335.

Bauman, Z. (1996). Tourists and vagabonds: heroes and victims of postmodernity. (Reihe Politikwissenschaft / Institut für Höhere Studien, Abt. Politikwissenschaft, 30). Wien: Institut für Höhere Studien (IHS), Wien. https://nbn- resolving.org/urn:nbn:de:0168-ssoar-266870.

Bauman, Z. (2000). Liquid Modernity. Cambridge: Polity.

Beerli, A., Díaz, G. \& Moreno, S. (2007). Self-congruity and destination choice. Annals of Tourism Research, 34(3), 571-587.

Bertaux, D. (1993). De la perspectiva de la historia de vida a la transformación de la práctica sociológica. Marinas, J. y Santamarina, C. La historia oral. Métodos y experiencias. Madrid: Ed. Debate.

Boissevain, J. (2005). Rituales ocultos. Protegiendo la cultura de la mirada turística. Pasos. Revista de turismo y patrimonio cultural, 3(2), 217-228.

Bowen, G. A. (2008). Naturalistic inquiry and the saturation concept: a research note. Qualitative research, 8(1), 137-152. 
Cáceres, P. (2008). Análisis cualitativo de contenido: una alternativa metodológica alcanzable. Psicoperspectivas. Individuo y sociedad, 2(1), 53-82.

Camprubí, R. \& Coromina, L. (2016). Content analysis in tourism research. Tourism Management Perspectives, 18, 134-140.

Cohen, E. (1974). Who is a tourist? A conceptual clarification. The sociological review, 22(4), 527-555.

Culler, J. (2007). Semiotics of tourism. The American Journal of Semiotics, 1(1/2), 127-140.

Doran, R., Larsen, S. \& Wolff, K. (2015). Different but similar: Social comparison of travel motives among tourists. International Journal of Tourism Research, 17(6), 555-563.

Elo, S. \& Kyngäs, H. (2008). The qualitative content analysis process. Journal of advanced nursing, 62(1), 107-115.

Festinger, L. (1954). A theory of social comparison processes. Human relations, 7(2), 117-140.

Franklin, A. (2003). The tourist syndrome: An interview with Zygmunt Bauman. Tourist studies, 3(2), 205-217.

Galí, N., Donaire, J. A., Martínez, E. \& Mundet, L. (2016). Turistas entre monumentos. El comportamiento de los visitantes en el centro histórico de Girona (2003 y 2013). RITUR-Revista Iberoamericana de Turismo, 5(2), 70-84.

Galí, N. \& Donaire, J. A. (2006). Visitors' Behavior in Heritage Cities: the case of Girona. Journal of Travel Research, 44(4), 442-448.

Hughes, H. L. (2002). Culture and tourism: a framework for further analysis. Managing Leisure, 7(3), 164-175.

Jacobsen, J. K. S. (2000). Anti-tourist attitudes: Mediterranean charter tourism. Annals of Tourism Research, 27(2), 284-300.

James, W. (1950). The Principles of Psychology, $3^{\text {rd }}$ edition, Dover Publications Inc., USA.

MacCannell, D. (1973). Staged authenticity: Arrangements of social space in tourist settings. American journal of Sociology, 79(3), 589-603.

Malhotra, N. K. (1988). Self concept and product choice: An integrated perspective. Journal of Economic Psychology, 9(1), 1-28.

Mayring, P. (2000). Qualitative content analysis. In Forum: Qualitative Social Research (Vol. 1, No. 2, p. 10).

McCabe, S. (2005). 'Who is a tourist?' A critical review. Tourist studies, 5(1), 85-106.

McCabe, S. \& Stokoe, E. H. (2004). Place and identity In tourist's accounts. Annals of Tourism Research, 31(3), 601-622.

McGehee, N. G. \& Andereck, K. L. (2004). Factors predicting rural residents' support of tourism. Journal of travel research, 43(2), 131-140.

McKercher, B. (2002). Towards a classification of cultural tourists. International journal of tourism research, 4(1), 29-38.

McKercher, B. (2004). A comparative study of international cultural tourists. Journal of Hospitality and Tourism Management, 11, 95-107.

McKercher, B. \& du Cros, H. (2003). Testing a cultural tourism typology. International Journal of Tourism Research, 5(1), 45-58.

Mejía, J. (2000). El muestreo en la investigación cualitativa. Investigaciones sociales, 4(5), 165-180.

Niemczyk, A. (2013). Cultural tourists:"An attempt to classify them". Tourism Management Perspectives, 5, 24-30.

Osti, L., Turner, L. W. \& King, B. (2009). Cultural differences in travel guidebooks information search. Journal of Vacation Marketing, 15(1), 63-78.

Phucharoen, P., Sriboonlue, U. \& Assarut, N. (2016). The effects of destination image, destination personality and self- congruity on tourists' intention. 14. 8991-9007.

Pizam, A. \& Sussmann, S. (1995). Does nationality affect tourist behavior? Annals of Tourism Research, 22(4), 901-917.

Plog, S. (1976). Why destination areas rise and fall in popularity. The Cornell hotel and restaurant administration quarterly, 55-58.

Prebensen, N. K., Larsen, S. \& Abelsen, B. (2003). I'm not a typical tourist: German tourists' self-perception, activities, and motivations. Journal of Travel research, 41(4), 416-420.

Richards, G. (1996). Production and consumption of European cultural tourism. Annals of tourism research, 23(2), 261-283. 
Richards, G. (2018). Cultural tourism: A review of recent research and trends. Journal of Hospitality and Tourism Management, 36, 12-21.

Richards, G. \& Russo, A. P. (2016). Synthesis and Conclusions: Towards a new geography of tourism?. En Antonio Paolo Russo y Greg Richards (Eds.) Reinventing the Local in Tourism: Producing, Consuming and Negotiating Place. Bristol: Channel View, pp. 251-266.

Rosenberg, M. (1979). Conceiving the Self. New York: Basic Books.

Silberberg, T. (1995). Cultural tourism and business opportunities for museums and heritage sites. Tourism management, 16(5), 361-365.

Sirgy, M. J. (1980). Self -concept in relation to product preference and purchase intention. In Developments in Marketing Science, Vol. 3, ed. V. V. Bellur, Marquette, MI: Academy of Marketing Science, 350-354.

Sirgy, M. J. (1982). Self-concept in consumer behavior: A critical review. Journal of consumer research, 9(3), 287-300.

Sirgy, M. J. \& Su, C. (2000). Destination image, self-congruity, and travel behavior: Toward an integrative model. Journal of Travel Research, 38(4), 340-352.

Swarbrooke, J. \& Horner, S. (2007). Consumer behaviour in tourism. Routledge.

Todd, S. (2001). Self-concept: A tourism application. Journal of Consumer Behaviour, 1(2), 184-196.

UNWTO (2008). International recommendations for tourism statistics.

UNWTO (2017). Definitions Committee on Tourism and Competitiveness (CTC).

UNWTO (2018). Report on Tourism and Culture Synergies. Madrid: UNWTO.

Woosnam, K. M., Draper, J., Jiang, J. K., Aleshinloye, K. D. \& Erul, E. (2018). Applying self-perception theory to explain residents' attitudes about tourism development through travel histories. Tourism Management, 64, 357-368.

Yeoman, I., Brass, D. \& McMahon-Beattie, U. (2007). Current issue in tourism: The authentic tourist. Tourism management, 28(4), 1128-1138.

Yu, X., Kim, N., Chen, C. C. \& Schwartz, Z. (2012). Are you a tourist? Tourism definition from the tourist perspective. Tourism Analysis, 17, 445-457. 